\title{
Communication Design of a Highly Miniaturized, Inherently Matched, Spherical Folded Dipole Antenna and Evaluation of its Quality Factor
}

\author{
Sanghamitro Das, Member, IEEE, Ashwin K. Iyer, Senior Member, IEEE
}

\begin{abstract}
A highly miniaturized three-dimensional spherical folded dipole antenna has been reported, for which inherent impedance matching is achieved with respect to a practical source impedance by employing a simple series $-L C$ loading combination, thereby engineering its input impedance. In order to maximize its bandwidth, the miniaturized antenna employs a spherical helix structure as the folded arm that occupies the full volume of the corresponding Chu sphere. A bifilar (two folded arms) and a quadrifilar (four folded arms) helix loaded folded dipole antenna are designed, and full-wave simulations show that both the resulting antennas demonstrate excellent impedance matching when miniaturized by $85 \%$ in comparison to a resonant dipole operating at the same frequency. Despite the high degree of miniaturization, the resulting radiation efficiencies for the bifilar and quadrifilar antennas are found to be $87.1 \%$ and $90.6 \%$, respectively. Furthermore, various quality-factor definitions are explored for the quadrifilar antenna, and it is observed that the resulting quality factor is around $1.83(1.22)$ times that predicted by the Chu (Thal) lower bound.
\end{abstract}

Index Terms-Electrically small antenna, folded dipole antenna, miniaturization, reactive loading, spherical helix, quality factor.

\section{INTRODUCTION}

Although the interest in electrically small antennas (ESAs) in the antenna community has grown significantly in recent years, the use of ESAs goes back as far as the late 1800s when Edison used vertical top-loaded grounded antennas for communicating between two distant points on land and/or sea, or Hertz performed his groundbreaking spark-gap experiment using a top-loaded dipole antenna; and in the early 1900s, when Marconi achieved trans-Atlantic communication using a fan-shaped monopole [1]. Today, ESAs are used extensively in a range of applications from broadcasting to mobile communications technologies like NFC, WiFi, WiMAX, MIMO etc., and sensing and identification applications like biomedical/environmental monitoring, machine-to-machine communications, body-centric applications, and RFID [2] - [8]. For most of these applications, dipole and monopole antennas along with their folded counterparts are often preferred, particularly due to their simple design and analysis, low cost, compact size, and well-defined radiation properties.

Despite being a popular choice for modern wireless systems, there exist well-known fundamental limits that put certain restrictions on the performance of an ESA in terms of its

Manuscript received Month XX, 202X; revised Month XX, 202X.

$\mathrm{S}$. Das is with the Department of Electrical and Computer Engineering, San Diego State University, San Diego, CA 92182, USA.

A. K. Iyer is with the Department of Electrical and Computer Engineering, University of Alberta, Edmonton, AB T6G 1H9, Canada (e-mail: iyer@ece.ualberta.ca). bandwidth and radiation efficiency [9]. The performance metrics of an ESA are usually linked to the electrical size $k a$ of the antenna [where, $k=2 \pi / \lambda$ is the wavenumber, $a$ is the radius of the smallest sphere circumscribing the antenna (also known as the Chu sphere), and $\lambda$ is the corresponding wavelength in free space] through the antenna's quality factor, $Q$, which is physically expected to have an inverse relationship with the antenna bandwidth [10]. As suggested by Chu and others [11] - [13], there exists a lower bound on the $Q$ factor, which in turn determines the maximum attainable bandwidth for an antenna of a given electrical size. It should also be noted that for an electrically small spherical wire-type antenna radiating like an electric dipole, which is similar to the miniaturized antenna reported here, the actual lower bound is approximately 1.5 times the Chu lower bound [14], and is typically known as the Thal lower bound. Nevertheless, with increasing miniaturization levels (i.e., decreasing electrical size), the $Q$ factor of the antenna increases, decreasing the maximum attainable bandwidth.

In order to achieve the maximum possible bandwidth, an ESA must occupy all the volume inside the corresponding Chu sphere. This is particularly a challenge for wire-type antennas such as dipoles. However, by realizing the dipole arm in the form of a spherical helix, one can maximize its bandwidth [15] - [17]. On the other hand, the process of miniaturization also results in a small input resistance and a large input reactance for the antenna, making it very difficult to match to a practical source impedance. This can typically be resolved by employing external matching networks, however at the expense of added loss and increased antenna footprint. In [15] - [17], the matching problem was resolved by using a multi-arm folded dipole antenna whose arms were realized in the shape of a spherical helix, and the miniaturized antennas were operated at their strongly reduced half-wavelength resonance frequencies. An alternative approach to realizing wide bandwidths without external matching networks involves the application of non-Foster or active loading elements in the antenna design [18] - [21]. While the antennas reported in [18] - [19] depend solely on non-Foster loading elements to achieve wide impedance matching bandwidth, the antennas described in [20] - [21] were initially designed for narrowband matching by manipulating the near-field coupling between the electrically small antennas and the metamaterial-inspired loading elements, and wide bandwidths were later realized by applying non-Foster loading techniques. Nevertheless, techniques involving non-Foster matching networks are typically limited to the lower-UHF frequency range (i.e., a few hundred megahertz), since achieving strong miniaturization at higher 
frequencies severely limits the available space for implementation of these active loading elements. Moreover, such active elements require an external dc bias and typically suffer from issues like stability, nonlinearity, and loss, which can also limit their use in several applications.

In [22] - [23], a mechanism was proposed for folded dipole antennas in order to achieve good inherent impedance matching at high miniaturization levels, without the need for either an external matching network or active/non-Foster loading; that is, the matching is inherent to the design of the (fully passive) antenna element. Unlike the technique described in [15] - [17], this technique makes use of the intrinsic first antiresonance of the folded dipole antenna that occurs naturally at a lower frequency than the half-wavelength resonance, and engineers the input impedance around it by applying simple series inductive and series capacitive loading to facilitate matching. Moreover, compared to the narrowband matching technique described in [20] - [21], this method is more intuitive and can be employed to match an electrically small folded dipole with respect to a wide range of source impedances. Besides, it was also shown that this technique could more generally be used to match complex source impedances, and thus is suitable for narrowband applications such as chip-based RFID [24]. However, since the miniaturized folded dipole antennas reported in [22] - [23] were not spherical in shape, there remains an opportunity to enhance their bandwidths using such an approach. Thus, this work develops a 3-D version of the folded dipole antenna presented in [23], where the folded arm has been realized in the shape of a spherical helix in order to maximize the bandwidth of the antenna, while the driven arm has been realized using a simple cylindrical shape loaded with two parallel plate capacitors in order to maintain inherent matching with a prescribed source impedance. The resulting antenna is $85 \%$ miniaturized relative to a half-wavelength dipole operating at the same frequency, and exhibits a quality factor close to the corresponding lower bound. A discussion is provided on how to estimate the $Q$ factor for the proposed antenna, and how it can be related to the corresponding antenna bandwidth.

This article is organized as follows. Section II describes the design principle and the structure of an $85 \%$ miniaturized bifilar folded dipole antenna, along with the simulated results. Section III discusses considerations for the evaluation of $Q$ of an equally miniaturized quadrifilar folded dipole antenna, and includes a comparison of its performance with respect to the corresponding lower bounds.

\section{Antenna Design And Simulation}

\section{A. Design Principle}

As described in [22], a folded dipole antenna is more amenable to inherent impedance matching at a high miniaturization level than a conventional dipole antenna, while exhibiting radiation performance comparable to its conventional counterpart miniaturized to the same level; this is because of the presence of an additional antiresonance before the conventionally used half-wavelength resonance. Although the antiresonance is not typically used due to very high input resistance, it was shown that the input impedance of the folded dipole antenna can be easily engineered around the antiresonance to yield an impedance equal to or approximately equal to the source impedance (with respect to which matching is to be done), just by applying a simple asymmetric loading combination of series inductive $(L)$ and series capacitive $(C)$ elements. Whereas the series inductive loading was responsible for providing the necessary miniaturization level by shifting the inherent resonances of the folded dipole towards lower frequencies, the series capacitive loading introduced a new resonance $f_{R, N}$ before the antiresonance $f_{A R, 1}$ of the folded dipole antenna, which is also the first inherent resonance of the antenna. Thereafter, by varying the inductive and capacitive loading values, the resonances $f_{R, N}$ and $f_{A R, 1}$ could be brought closer to each other, and their mutual positions could be adjusted in order to achieve the desired $\Re\left\{Z_{i n}\left(f_{R, N}\right)\right\}$. This results in impedance matching at $f_{R, N}$, which is accomplished inherently by the abovementioned design technique and without an external matching network.

It was also shown in [22] that the currents on the folded dipole arm are directed opposite to those on the driven arm, unlike the examples given in [17] where these currents are co-directed and radiate in phase. However, due to the fact that the magnitude of currents on the folded arm was much higher than that on the driven arm, there occurred a net current imbalance, which afforded high radiation efficiency. Clearly, the radiation efficiencies for the antenna structures reported in [17] were much higher $(>90 \%)$ compared to the radiation efficiency reported in [22] $(72.3 \%)$. However, it must be noted that achieving inherent impedance matching for arbitrary miniaturization levels and source impedances is more flexible in the approach of [22] than that of [17]. This can be explained by studying the different current components of a folded dipole antenna. It is well known that the overall current on the folded dipole arms can be decomposed into two components: (1) a loop-like or contra-directed current component [also known as the differential mode, odd mode, or transmission-line mode (TLM)], and (2) a co-directed current component [also known as the common mode, even mode, or antenna mode (AM)] that accounts for most of the radiation. While the TLM current does not contribute strongly to radiation, both TLM and AM currents do influence the input impedance of the antenna, and thus both are required to efficiently match the antenna.

The highly miniaturized and inherently matched spherical folded dipole antennas reported in this article are designed following the same working principle described in [22], and as mentioned earlier, are an evolution of the fully printed, planar, and highly miniaturized inherently matched folded dipole antenna reported in [23]. It is designed to exhibit a wider bandwidth at a lower miniaturization level and possesses a higher co-to-cross-polarization separation owing to its unique geometry; these attributes will be demonstrated in the next subsection.

\section{B. Antenna Structure}

The different stages of the antenna construction are displayed in Fig. 1. In the first stage, the driven arm is constructed 


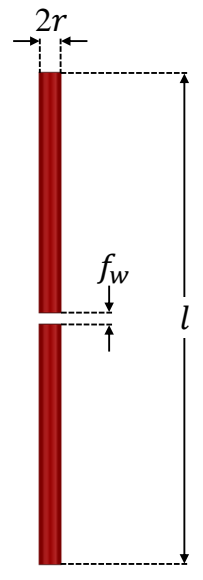

(a)

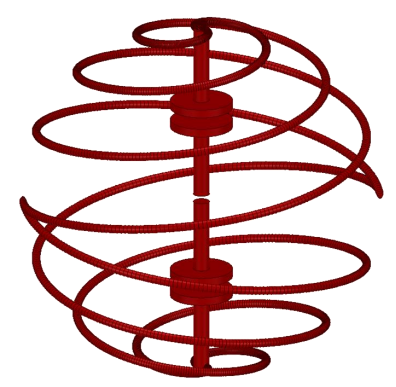

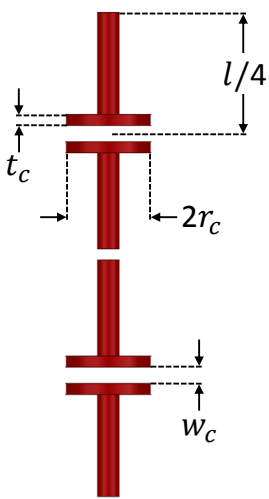

(b)

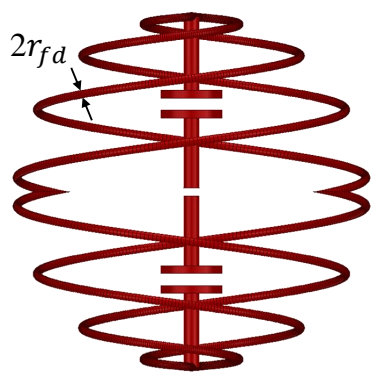

(c)

Fig. 1. Step-by-step construction of the miniaturized spherical helix loaded folded dipole antenna: (a) cylindrical driven arm, (b) application of capacitive loading, and (c) complete antenna structure with both inductive and capacitive loading (the left-hand-side image shows the antenna in profile, whereas the right-hand-side image shows the antenna's E-plane cross-section).

by realizing an electrically small cylindrical dipole antenna of length $l$ and radius of cross-section $r$. The cylindrical driven arm is shown in Fig. 1(a). In the next phase, parallel-plate capacitors are introduced on the cylindrical driven arm at a distance $l / 4$ from the antenna ends, as shown in Fig. 1(b), following the loading-capacitance locations used in [22] [23]. The individual parallel plate is circular in shape, with a radius $r_{c}$ and a thickness $t_{c}$. The gap between the parallel plate $w_{c}$ together with the plate radius $r_{c}$ determine the amount of series capacitive loading. In the final phase, spherical helical folded arms are added between the ends of the antenna in order to produce the required distributed inductive loading for miniaturization. While the radius of the spherical helix $(l / 2)$ is limited by the overall dimension of the antenna, the number of turns can be optimized in order to realize the required loading inductance. It was shown in [17] that the bandwidth of a folded spherical helix antenna can be improved by increasing the number of folded helical arms. Although the design shown in Fig. 1 admits an arbitrary number of folded arms, an excessive number of arms may cause difficulties in feeding the antenna, as the feeding circuit needs to extend through the spherical helix into the centre of the antenna. Thus,
TABLE I

OPTIMIZED DIMENSIONS FOR THE MINIATURIZED SPHERICAL HELIX LOADED FOLDED DIPOLE ANTENNA

\begin{tabular}{cc}
\hline Dimension & Value \\
\hline$l$ & $44 \mathrm{~mm}$ \\
\hline$r$ & $1 \mathrm{~mm}$ \\
\hline$f_{w}$ & $1 \mathrm{~mm}$ \\
\hline$r_{c}$ & $2.74 \mathrm{~mm}$ \\
\hline$t_{c}$ & $1 \mathrm{~mm}$ \\
\hline$w_{c}$ & $1.85 \mathrm{~mm}$ \\
\hline$r_{f d}$ & $0.5 \mathrm{~mm}$ \\
\hline Number of helix turns & 4.16
\end{tabular}

for simplicity and practicality of excitation, a bifilar case (i.e., two folded arms with a $180^{\circ}$ angular separation) is presented. Later in this work, a quadrifilar case (i.e., four folded arms with a $90^{\circ}$ angular separation) is also presented for the purpose of comparing its quality factor with that of the equivalently miniaturized folded spherical helix antenna reported in [17]. Finally, as shown in Fig. 1(c), the sense of rotation of the helical folded arm is made to change halfway between the antenna ends in order to reduce cross-polarized radiation in the broadside direction $\left(\theta=90^{\circ}\right)$. The overall length of the antenna $l$ was chosen to be $44 \mathrm{~mm}$, and the other dimensions were optimized for operation at an $85 \%$ miniaturization level (i.e., at $515 \mathrm{MHz}$ where $l \approx 0.075 \lambda$ ) compared to an unloaded slender half-wavelength dipole antenna operating at the same frequency. The optimized antenna dimensions are given in Table I.

\section{Simulated Results}

While a miniaturized folded dipole antenna can be designed for matching with respect to practically any source impedance less than $\Re\left\{Z_{i n}\left(f_{A R, 1}\right)\right\}$ using the technique described in [22], the source impedance for the antenna reported in this article was selected to be $100 \Omega$. Simulations were performed in Ansys HFSS, where bulk copper was used as the conducting material for the antenna, and no additional losses (e.g., surface roughness) were introduced. For the dimensions listed in Table I, the spherical folded dipole antenna produced very good return loss of $41.6 \mathrm{~dB}$ at $515 \mathrm{MHz}$, with $k a=0.237$. The input impedance and $S_{11}$ of the optimized antenna are shown in Figs. 2(a) and 2(b), respectively, and the corresponding 3-dB and 10-dB return-loss bandwidths of the antenna were found to be $7.41 \mathrm{MHz}(\sim 1.44 \%)$ and $2.42 \mathrm{MHz}(\sim 0.47 \%)$, respectively.

The E-plane and H-plane gain patterns are shown in Figs. 3(a) and 3(b), respectively. It can be seen from Fig. 3 that the broadside gain of the antenna at $515 \mathrm{MHz}$ is $1.28 \mathrm{dBi}$ and, although not observable in the scale shown, the co-to-crosspolarization separation is $82.3 \mathrm{~dB}$ at broadside. The radiation efficiency of the antenna at $515 \mathrm{MHz}$ was found to be $87.1 \%$. Finally, the current distribution of the antenna is shown in Fig. 4. As can be seen, most of the radiation at the operating frequency is owed to the currents on the helical folded arm, similar to the case described in [22]. 


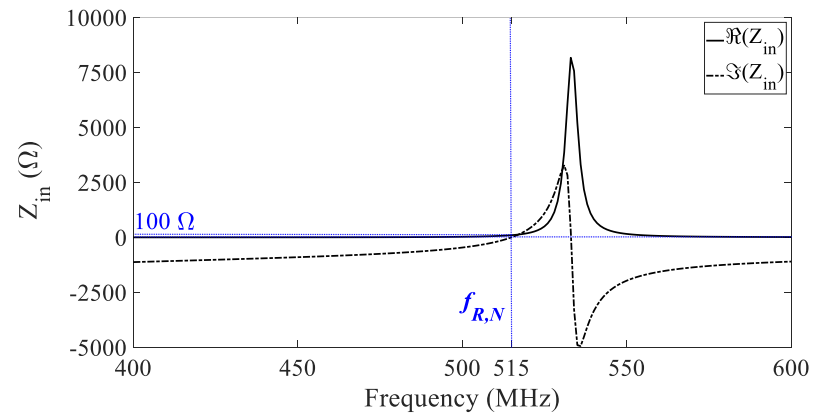

(a)

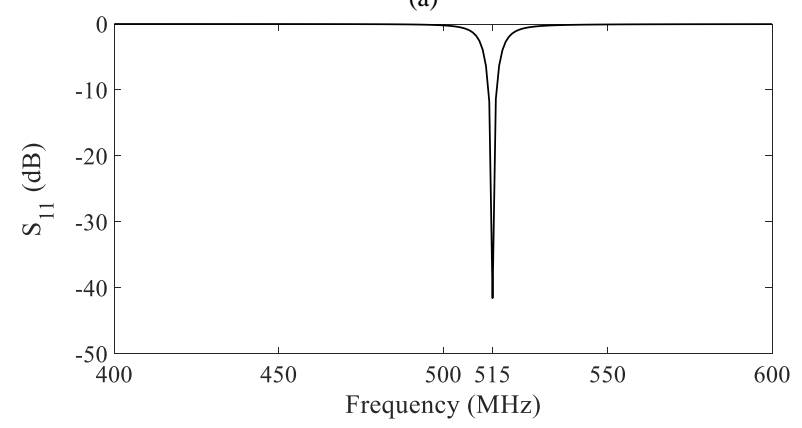

(b)

Fig. 2. (a) Input impedance and (b) $\mathrm{S}_{11}$ of the spherical helix loaded folded dipole antenna.

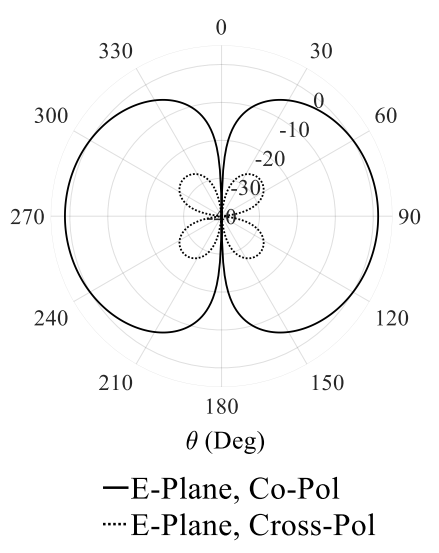

(a)

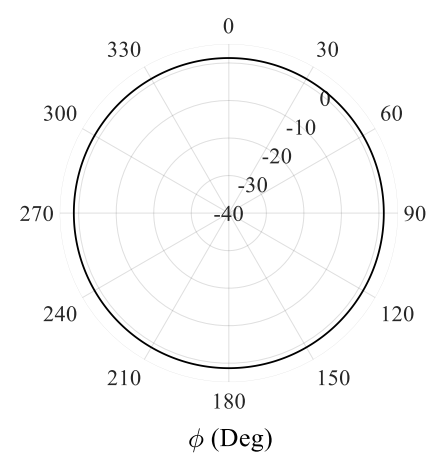

-H-Plane, Co-Pol

(b)
Fig. 3. Gain patterns at $515 \mathrm{MHz}$ (a) E-plane and (b) H-plane (Note: Only copol pattern is shown for the $\mathrm{H}$-plane as the cross-pol remains below $-80 \mathrm{~dB}$ and would not be visible in the scale used).

\section{ESTIMATION OF QUALity FACTOR}

The $Q$ factor of an antenna is a valuable metric that connects the electrical size of the antenna, $k a$, to its bandwidth and radiation efficiency, and it is particularly useful for describing the performance of an ESA. For a given electrical size, expressions can be found for the $Q$ lower bound, which in turn determines the maximum attainable bandwidth for the antenna. For a generally lossy antenna with a radiation efficiency of $\eta_{\text {rad }}$, the Chu lower bound of $Q$ (denoted hereafter as $Q_{l b, C h u}$ ) can be given as [16]:

$$
Q_{l b, C h u}=\eta_{\text {rad }}\left[\frac{1}{k a}+\frac{1}{(k a)^{3}}\right] .
$$

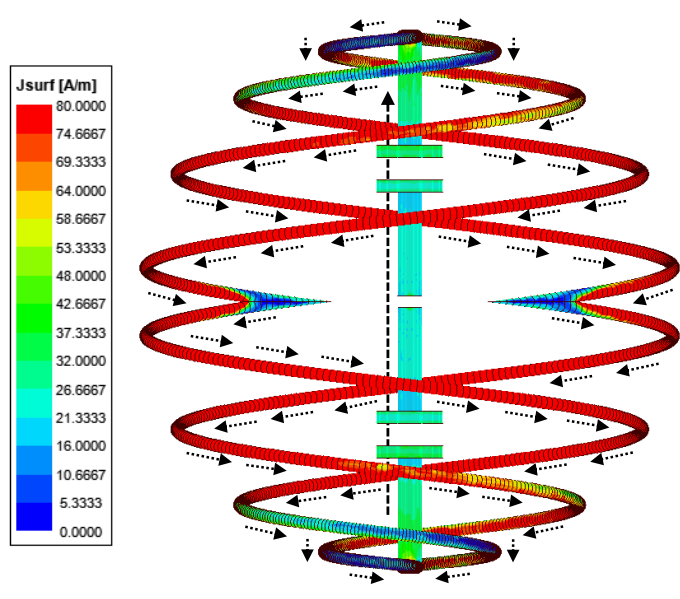

Fig. 4. Current distribution on the spherical helix loaded folded dipole antenna at $515 \mathrm{MHz}$ (arrows indicate the direction of the current flow on the cylindrical driven arm and the helical folded arms).

Numerous approaches exist in the literature for estimating the $Q$ of an ESA; however, arguably the most widely used expressions for determining $Q$ are given by Yaghjian and Best [11]. Three definitions can be found in [11] for the quality factor of an antenna, the first of which is the $Q$ determined from antenna fields:

$$
\begin{aligned}
Q_{F}\left(\omega_{0}\right)= & \mid \frac{\omega_{0}}{2 R_{\text {in }}\left(\omega_{0}\right)} X_{i n}^{\prime}\left(\omega_{0}\right) \\
& \quad-\frac{2 \omega_{0}}{\left|I_{0}\right|^{2} R_{i n}\left(\omega_{0}\right)}\left[W_{\mathcal{L}}\left(\omega_{0}\right)+W_{\mathcal{R}}\left(\omega_{0}\right)\right] \mid
\end{aligned}
$$

where $\omega_{0}$ is the operating frequency, $R_{i n}\left(\omega_{0}\right)$ and $X_{i n}\left(\omega_{0}\right)$ are the real and imaginary parts of the input impedance $Z_{i n}$ at the operating frequency, respectively, and $X_{i n}^{\prime}\left(\omega_{0}\right)$ is the frequency derivative of $X_{i n}\left(\omega_{0}\right) . W_{\mathcal{L}}$ and $W_{\mathcal{R}}$ are dispersion energies that are directly related to the lost and radiated powers, respectively, and are calculated using the fields and currents of the antenna. The second definition of quality factor is given entirely in terms of the input impedance of the antenna:

$$
\begin{aligned}
& Q_{Z}\left(\omega_{0}\right)=\frac{\omega_{0}}{2 R_{i n}\left(\omega_{0}\right)}\left|Z_{i n}^{\prime}\left(\omega_{0}\right)\right| \\
& =\frac{\omega_{0}}{2 R_{i n}\left(\omega_{0}\right)} \sqrt{\left[R_{i n}^{\prime}\left(\omega_{0}\right)\right]^{2}+\left[X_{i n}^{\prime}\left(\omega_{0}\right)+\frac{X_{i n}\left(\omega_{0}\right)}{\omega_{0}}\right]^{2}}
\end{aligned}
$$

where once again primes indicate frequency derivatives. The final definition of quality factor relates directly to the fractional bandwidth $F B W$ of the antenna for a given VSWR level $s$, and is given as

$$
Q_{B W}\left(\omega_{0}\right)=\frac{2 \sqrt{\beta}}{F B W\left(\omega_{0}\right)}, \quad \sqrt{\beta}=\frac{s-1}{2 \sqrt{s}}
$$

All the three quality factors are independent of whether they are estimated at the resonance or the antiresonance of the antenna, and are approximately equal for an antenna tuned at its resonance/antiresonance that does not have another resonance/antiresonance within its defined VSWR bandwidth. Furthermore, the quality factors exhibit an inverse relationship 

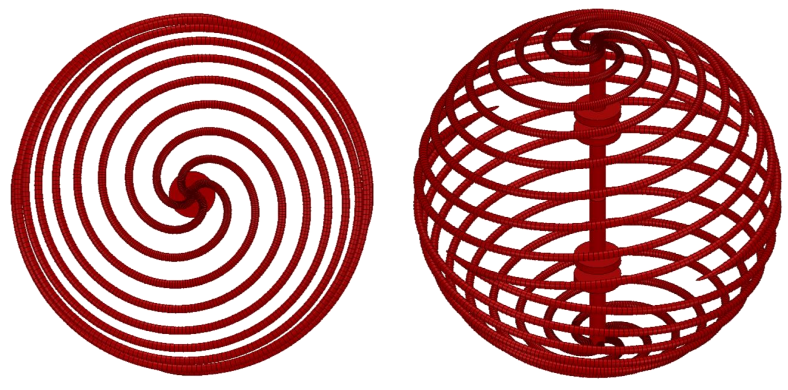

(a)

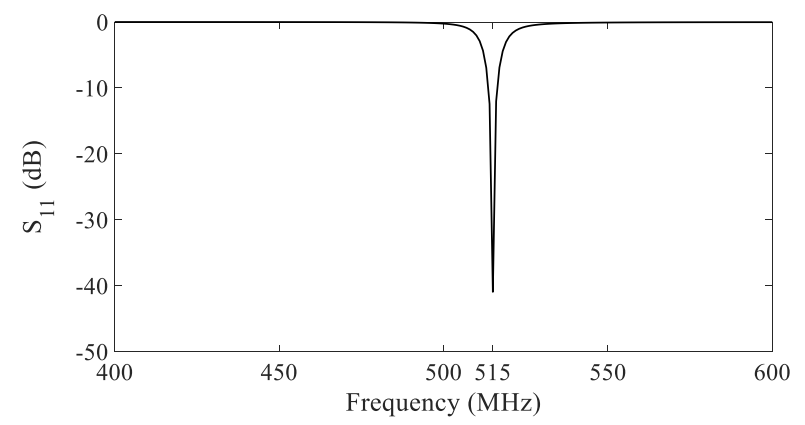

(b)

Fig. 5. (a) Miniaturized quadrifilar spherical helix loaded folded dipole antenna, $l=44 \mathrm{~mm}, r=1 \mathrm{~mm}, f_{w}=1 \mathrm{~mm}, r_{c}=2.88 \mathrm{~mm}, t_{c}=1 \mathrm{~mm}, w_{c}=$ $1.85 \mathrm{~mm}, r_{f d}=0.5 \mathrm{~mm}$, and number of helix turns $=4.5$, (b) corresponding $\mathrm{S}_{11}$ with respect to $100 \Omega$ source impedance.

with the bandwidth, which is arguably the most important property of the quality factor of a system. However, this consistency among the different $Q$ factor definitions and their inverse proportionality with bandwidth fall apart for the case of a multi-resonant antenna, where multiple resonances are employed in order to enhance bandwidth. In [25], it was shown that for such a multi-resonant antenna, the three quality factors are no longer comparable near the closely-spaced resonances, and although $Q_{F}$ does not violate the lower bound, it is no longer inversely related to the bandwidth, which raises a question about the validity of $Q_{F}$ for these cases. On the other hand, despite $Q_{Z}$ violating the lower bound, it continues to demonstrate an inverse relationship with bandwidth, and it remains comparable with $Q_{B W}$ for a small enough VSWR level, such that the defined bandwidth does not contain more than one resonance/antiresonance [25] - [27]. Nevertheless, for the miniaturized spherical helix loaded folded dipole antenna described here, no such issues arise, since the matched resonance $f_{R, N}$ is sufficiently isolated from the neighbouring antiresonance $f_{A R, 1}$ due to the narrow bandwidth of the antenna.

A quadrifilar version of the proposed miniaturized antenna was designed in order to make a fairer comparison of qualityfactor performance versus the folded spherical helix antenna described in [17], which has a comparable $k a$ value and four helical arms. The dimension $r_{c}$ and number of helix turns of the proposed quadrifilar antenna were adjusted slightly in order to maintain impedance matching with respect to a $100-\Omega$ source impedance at the same $515 \mathrm{MHz}$ operating frequency (i.e., $85 \%$ miniaturization level). The quadrifilar

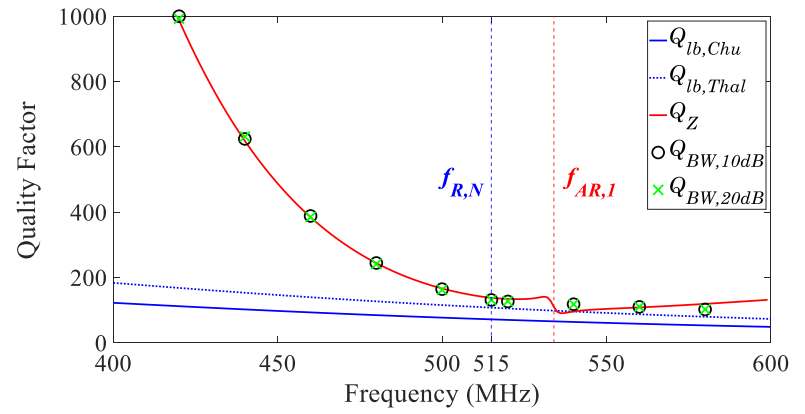

Fig. 6. Comparison of quality factors for the miniaturized quadrifilar spherical helix loaded folded dipole antenna. The vertical dashed lines mark the positions of the matched resonance $f_{R, N}$ and the neighbouring antiresonance $f_{A R, 1}$.

structure is depicted in Fig. 5(a), along with the corresponding $\mathrm{S}_{11}$ response in Fig. 5(b). The relevant dimensions re are given in the figure caption. The resulting $3-\mathrm{dB}$ and $10-\mathrm{dB}$ returnloss bandwidths are now $8 \mathrm{MHz}(\sim 1.55 \%)$ and $2.63 \mathrm{MHz}$ $(\sim 0.51 \%)$, respectively, which are wider compared to that of the bifilar case described in the previous section. This result is analogous to that observed by Best and Hanna in [17]. The corresponding radiation efficiency and gain at $515 \mathrm{MHz}$ are $90.6 \%$ and $1.46 \mathrm{dBi}$, respectively, which are higher compared to the bifilar case, mainly due to the larger number of codirected current carrying helical arms for the quadrifilar case. By comparison, the $83 \%$ miniaturized $(k a=0.265)$ quadrifilar folded spherical helix antenna reported in [17] exhibits $2.37 \%$ $3-\mathrm{dB}$ bandwidth and $97.4 \%$ radiation efficiency.

A graph was produced to compare $Q_{Z}$ and $Q_{B W}$ with $Q_{l b, C h u}$ over the whole frequency range. While $Q_{l b, C h u}$ and $Q_{Z}$ may be easily estimated over a range of frequencies using (1) and (3), respectively, one must match the antenna at each of the frequency points in order to obtain the correct $Q_{B W}$ and estimate the corresponding bandwidths from the $\mathrm{S}_{11}$ response at the chosen VSWR levels. In this work, matching was obtained at select frequencies over the band using a unique (and fully reactive) L-section matching network designed for each frequency in order to ensure that the source impedance could be maintained at $100 \Omega$ (of course, no such matching network is needed at the antenna operating frequency of $f_{R, N}=515 \mathrm{MHz}$ ). As mentioned earlier, the actual quality-factor lower bound for an electrically small spherical electric-dipole-like antenna is given by the Thal lower bound (hereafter denoted as $Q_{l b, T h a l}$ ), which is approximately 1.5 times $Q_{l b, C h u}$. Thus, a comparison of the different quality factors, including both the $\mathrm{Chu}$ and Thal lower bounds for the designed quadrifilar antenna is shown in Fig. 6. As can be seen, $Q_{Z}$ remains approximately equal to $Q_{B W}$ for the chosen VSWR levels, i.e., $s=1.9(10-\mathrm{dB}$ return loss) and $s=1.2$ (20-dB return loss), and as expected, none of them violates the Chu lower bound. On the other hand, both $Q_{Z}$ and $Q_{B W}$ are very close to the Thal lower limit $(\sim 1.22$ times $)$ near the frequency of operation.

In Table II, the quality factors for the proposed quadrifilar miniaturized antenna are compared with those of the folded spherical helix antenna described in [17] at their respective 
TABLE II

CALCULATION OF $Q$ FACTORS FOR MINIATURIZED SPHERICAL HELIX LOADED DIPOLE ANTENNAS

\begin{tabular}{cccccc}
\hline Antenna & $k a$ & $\eta_{r}$ & $\begin{array}{c}Q_{l b} \\
\text { (including } \\
\text { losses) }\end{array}$ & $Q_{Z}$ & $Q_{Z} / Q_{l b}$ \\
\hline \hline $\begin{array}{c}\text { Quadrifilar } \\
\text { folded spherical } \\
\text { helix from [17] }\end{array}$ & 0.265 & $97.4 \%$ & 55.6 & 84.64 & 1.52 \\
\hline $\begin{array}{c}\text { Proposed } \\
\text { quadrifilar folded } \\
\text { spherical helix }\end{array}$ & 0.237 & $90.6 \%$ & 71.65 & 130.9 & 1.83 \\
\hline
\end{tabular}

operating points. It can be seen that, although the resulting quality factor for the quadrifilar case described here is very close to the $Q$ lower bounds, it does not outperform the antenna reported in [17]. However, it should be noted that the proposed quadrifilar antenna is $9 \%$ smaller with respect to the antenna from [17] (i.e., $k a=0.237$ versus $k a=0.265$ ). Nevertheless, as mentioned earlier, the impedance matching mechanism reported in this work is much more robust.

\section{CONCLUSION}

In this work, an alternative technique for achieving inherent impedance matching has been demonstrated for a threedimensional highly miniaturized folded dipole antenna, while simultaneously maximizing the bandwidth by employing an overall spherical shape. The technique enables matching with respect to a wide range of source impedances, at any given miniaturization level. The designed bifilar and quadrifilar antennas, which are $85 \%$ miniaturized compared to an unloaded resonant dipole operating at the same frequency, exhibit bandwidths close to that of an equivalently miniaturized spherical folded dipole antenna reported in the literature, while exhibiting $87.1 \%$ and $90.6 \%$ radiation efficiencies, respectively. Two quality factors, $Q_{Z}$ and $Q_{B W}$, were computed for the quadrifilar antenna and compared with the corresponding lower bounds $Q_{l b, C h u}$ and $Q_{l b, T h a l}$. As expected, $Q_{Z}$ was found to be approximately equal to $Q_{B W}$ for the selected VSWR levels. It was also observed that both $Q_{Z}$ and $Q_{B W}$ do not violate the Chu lower bound and are very close to the Thal lower bound around the frequency of operation.

Owing to the global pandemic situation, the challenging fabrication and measurement of the proposed antenna could not be performed. We expect to report such results in a future communication, once access to resources has been restored.

\section{ACKNOWLEDGMENT}

The authors would like to thank Dr. Arthur D. Yaghjian for insightful discussions regarding the estimation of $Q$ factors and CMC Microsystems for providing the required simulation tools.

\section{REFERENCES}

[1] J. Ramsay, "Highlights of Antenna History," IEEE Antennas Propag. Soc. Newslett., vol. 23, no. 6, pp. 7-20, Dec. 1981.

[2] K. Fujimoto and H. Morishita, Modern Small Antennas. NY, USA: Cambridge University Press, 2013.
[3] K. S. Nikita, Handbook of Biomedical Telemetry. Hoboken, NJ, USA: John Wiley \& Sons, Inc., 2014.

[4] S. Rao, N. Llombart, E. Moradi, K. Koski, T. Björninen, L. Sydänheimo, J. M. Rabaey, J. M. Carmena, Y. R.-Samii, and L. Ukkonen, "Miniature Implantable and Wearable On-Body Antennas: Towards the New Era of Wireless Body-Centric Systems," IEEE Antennas Propag. Mag., vol. 56, no. 1, pp. 271-291, Feb. 2014.

[5] L. Lizzi and F. Ferrero, "Use of Ultra-Narrow Band Miniature Antennas for Internet-of Things Application," Electron. Lett., vol. 51, no. 24, pp. 1964-1966, Nov. 2015.

[6] Y. Gao, R. Ma, Q. Zhang, and C. Parini, "UHF Antennas for Machineto-Machine Communications and Internet of Things," in Proc. 10th Eur. Conf. Antennas Propag. (EuCAP), Davos, pp. 1-3, Apr. 2016.

[7] T.-W. Koo, Y.-J. Hong, G.-K. Park, K. Shin, and J.-G. Yook, "Extremely Low-Profile Antenna for Attachable Bio-Sensors," IEEE Trans. Antennas Propag., vol. 63, no. 4, pp. 1537-1545, Apr. 2015.

[8] G. Marrocco, "The Art of UHF RFID Antenna Design: Impedancematching and Size-Reduction Techniques," IEEE Antennas Propag. Mag., vol. 50, no. 1, pp. 67-79, Feb. 2008.

[9] R. C. Hansen, "Fundamental Limitations in Antennas," Proc. IEEE, vol. 69, no. 2, pp. 170-182, Feb. 1981.

[10] A. D. Yaghjian and S. R. Best, "Impedance, Bandwidth, and Q of Antennas," IEEE Trans. Antennas Propag., vol. 53, no. 4, pp. 12981324, Apr. 2005.

[11] L. J. Chu, "Physical Limitations on Omni-Directional Antennas," $J$. Appl. Phys., vol. 19, no. 12, pp. 1163-1175, Dec. 1948.

[12] R. Collin and S. Rothschild, "Evaluation of Antenna Q," IEEE Trans. Antennas Propag., vol. 12, no. 1, pp. 23-27, Jan. 1964.

[13] J. S. McLean, "A Re-Examination of the Fundamental Limits on the Radiation $Q$ of Electrically Small Antennas," IEEE Trans. Antennas Propag., vol. 44, no. 5, pp. 672-676, May 1996.

[14] H. L. Thal, Jr., "New Radiation $Q$ Limits for Spherical Wire Antennas," IEEE Trans. Antennas Propag., vol. 54, no. 10, pp. 2757-2763, Oct. 2006.

[15] S. R. Best, "The Radiation Properties of Electrically Small Folded Spherical Helix Antennas," IEEE Trans. Antennas Propag., vol. 52, no. 4, pp. 953-960, Apr. 2004.

[16] S. R. Best, "Low $Q$ Electrically Small Linear and Elliptical Polarized Spherical Dipole Antennas," IEEE Trans. Antennas Propag., vol. 53, no. 3, pp. 1047-1053, Mar. 2005.

[17] S. R. Best and D. L. Hanna, "A Performance Comparison of Fundamental Small-Antenna Designs," IEEE Antennas Propag. Mag., vol. 52, no. 1, pp. 47-70, Feb. 2010.

[18] H. Mirzaei and G. V. Eleftheriades, "A Resonant Printed Monopole Antenna with an Embedded Non-Foster Matching Network," IEEE Trans. Antennas Propag., vol. 61, no. 11, pp. 5363-5371, Nov. 2013.

[19] E. A. Elghannai, B. D. Raines, and R. G. Rojas, "Multiport Reactive Loading Matching Technique for Wide Band Antenna Applications Using the Theory of Characteristic Modes," IEEE Trans. Antennas Propag., vol. 63, no. 1, pp. 261-268, Jan. 2015.

[20] P. Jin and R. W. Ziolkowski, "Broadband, Efficient, Electrically Small Metamaterial-Inspired Antennas Facilitated by Active Near-Field Resonant Parasitic Elements," IEEE Trans. Antennas Propag., vol. 58, no. 2, pp. 318-327, Feb. 2010.

[21] M. Barbuto, A. Monti, F. Bilotti, and A. Toscano, "Design of a NonFoster Actively Loaded SRR and Application in Metamaterial-Inspired Components," IEEE Trans. Antennas Propag., vol. 61, no. 3, pp. 12191227, Mar. 2013.

[22] S. Das, D. J. Sawyer, N. Diamanti, A. P. Annan, and A. K. Iyer, "A Strongly Miniaturized and Inherently Matched Folded Dipole Antenna for Narrowband Applications," IEEE Trans. Antennas Propag., vol. 68, no. 5, pp. 3377-3386, May 2020.

[23] S. Das and A. K. Iyer, "Fully Printed and Electrically Small Folded Dipole with Inherent Matching," in Proc. IEEE Antennas Propag. Soc. Int. Symp., Montréal, QC, Canada, Jul. 2020, pp. 389-390.

[24] S. Das, H. Saghlatoon, P. Mousavi, and A. K. Iyer, "A Highly Miniaturized and Inherently Conjugately Matched Folded Dipole-Based RFID Tag Antenna," IEEE Access, vol. 7, pp. 101658-101664, Jul. 2019.

[25] H. R. Stuart, S. R. Best, and A. D. Yaghjian, "Limitations in Relating Quality Factor to Bandwidth in a Double Resonance Small Antenna," IEEE Antennas Wireless Propag. Lett., vol. 6, pp. 460-463, 2007.

[26] S. R. Best, "The Inverse Relationship between Quality Factor and Bandwidth in Multiple Resonant Antennas," in Proc. IEEE Antennas Propag. Soc. Int. Symp., Albuquerque, NM, USA, Jul. 2006, pp. 623-626.

[27] S. R. Best, "Bandwidth and Lower bound on Q for Small Wideband Antennas," in Proc. IEEE Antennas Propag. Soc. Int. Symp., Albuquerque, NM, USA, Jul. 2006, pp. 647-650. 